\title{
Calculating printing speed for polylactic acid continuous glass fiber composites via fused filament fabrication
}

\author{
Behnam Akhoundi ${ }^{1}$, Mojtaba Nabipour ${ }^{1}$, Omid Kordi ${ }^{1}$, Faramarz Hajami ${ }^{2}$, Shahab S. Band $3,4^{*}$ \\ and Amirhosein Mosavi 5,6,7* \\ 1 Additive Manufacturing Laboratory, Faculty of Mechanical Engineering, Tarbiat Modares University, \\ Jalaal-e Al-Ahmed ExpWay, Tehran 14115-143, Behnam.Akhoundi@modares@modares.ac.ir; \\ Mojtaba.Nabipour@modares.ac.ir; Omid_Kordi@modares.ac.ir \\ 2 Department of Mechanical Engineering, Faculty of Mechatronics, Karaj Branch, Islamic Azad University, \\ Karaj, Iran, faramarz.hajami@kiau.ac.ir \\ 3 Institute of Research and Development, Duy Tan University, Da Nang 550000, Vietnam. \\ 4 Future Technology Research Center, College of Future, National Yunlin University of Science and \\ Technology 123 University Road, Section 3, Douliou, Yunlin 64002, Taiwan, R.O.C \\ 5 Faculty of Civil Engineering, Technische Universität Dresden, 01069 Dresden, Germany \\ 6 School of Economics and Business, Norwegian University of Life Sciences, 1430 Ås, Norway \\ 7 Kando Kalman Faculty of Electrical Engineering, Obuda University, 1034 Budapest, Hungary \\ * Correspondence: amir.mosavi@mailbox.tu-dresden.de; shamshirbandshahaboddin@duytan.edu.vn
}

\begin{abstract}
In this study, a novel task of printing speed optimization for continuous fiber composites is investigated. Using continuous fibers is an innovative approach to reinforce products made by fused filament fabrication (FFF) additive manufacturing (AM) technology. In the printing process of composites with continuous fibers, the printing speed is critical because of its significant effect on the geometric shape of the samples, especially their corners. During optimization in this research, continuous glass fiber (CGF) and polylactic acid (PLA) filaments were utilized as reinforcing phase and matrix, respectively, and were simultaneously fed into the extrusion-based polymer 3D printer to form PLA/CGF composites. The optimization was carried out by calculating the temperature changes of the deposited rasters in the presence and absence of fibers as a first step and then determining the special relationship between the printing speeds and rasters temperature changes. Finally, the optimal and the maximum printing speed was computed based on a hypothesis, which is proved by the results of high-quality printed composites with different geometric shapes.
\end{abstract}

Keywords: optimization; extrusion-based additive manufacturing; $3 \mathrm{~d}$ printer; continuous fibers

\section{Introduction}

Additive Manufacturing (AM) is a suitable name used to describe the technologies that make 3D objects by adding layer-upon-layer of material, such as metals, plastics, ceramics etc. Various methods are available for printing polymeric products like stereolithography and digital light processing for liquid photopolymers, fused filament fabrication (FFF) for polymer filaments, selective laser sintering method for polymeric powders and laminated object manufacturing for polymer sheets [1-3]. Among all methods, FFF is one of the most commonly used 3D printing technologies with different industries due to low cost and easily accessible [4,5]. The production of very complex shapes, less waste generation, high flexibility, no need for jigs and fixtures and changing materials with relative ease are other great advantages. Also, it has entered many areas of applications such as medicine, dentistry, aerospace, food industry, consumer products, etc. [6-9]. 
The main challenge in manufacturing parts with FFF process is the low mechanical properties of products due to the nature of thermoplastic polymers in comparison with metallic and ceramic materials [3,10]. The mechanical properties of FFF samples are usually weaker than other parts, which are made by plastic injection molding or compression molding. In those traditional processes, materials are shaped under pressure to form final parts, but during FFF process a sample is produced by bonding rasters and layers together without any external pressure or compression [11-14]. Therefore, improving the mechanical properties of FFF samples has always been important for researchers to achieve final parts with better mechanical properties $[7,15,16]$.

The most effective way to dramatically increase the tensile mechanical properties is utilizing continuous fibers, which affect the tensile strength and modulus of final products [17,18]. Extensive researches have been done on 3D printing of continuous fiber composites and their main focus was on experimental evaluation of mechanical properties of 3D printed products. Most of the printed products had low quality with simple or shell-like geometric shapes [19].

Based on previous studies, Mori et al. [20] improved the tensile strength of acrylonitrile butadiene styrene (ABS) products approximately four times, with $1.4 \%$ volumetric continuous carbon fiber (CCF) and a heat-treatment process. Printed specimens were standard specimens of tensile and fatigue tests (dog-bone), and their surface quality was low. Van der klift et al. [21] employed a Mark One 3D printer from Markforged company and investigated the tensile strength of polyamide (PA)/CCF composites. Matsuzaki et al. [22] utilized polylactic acid (PLA)/CCF and PLA/Jute composites by the simultaneous impregnation system to evaluate the mechanical properties of composite samples. Printed specimens were standard specimens of the tensile test (dog-bone) and rectangular shape, and were undesirable in appearance, dimensional accuracy and surface quality. Tian et al. [23] employed the simultaneous impregnation method to print PLA/CCF and evaluated the effect of process parameters on the flexural strength modulus of composites. Printed samples were in the shell form. They examined the layer height, nozzle temperature, feed of filament, bead width, and printing speed at different levels. It should be noted that the effect of the mentioned parameters on mechanical properties was predictable. In the light of nozzle temperature, as the melt flow index increased, fibers' impregnation with polymeric materials was more favorable and the bonding between layers and rasters for the matrix phase was also higher; therefore, the strength of the composite samples improved. Moving on layer height, fibers' diameter was constant, but as the layer height increased, the strength of the composites became weaker due to the reduction in fiber percentage. In case of the filament feed, the lower rate meant better impregnation, leading to a higher composite strength. For bead width, the effect was similar to the layer height. With the reduction in bead width, there was an increase in the fiber percentage, improving the mechanical properties. The effect of the printing speed is similar to filament feeding rate and the low speed resulted in better impregnation with increasing the composite strength [19].

Malenka et al. [24] employed a Mark One 3D printer and evaluated the tensile modulus of PA/Kevlar composite samples. Printed specimens were standard specimens of the tensile test (dog-bone), and the radius of the fibers at the corners was clear. Li et al. [25] improved the bonding between fibers and matrix of PLA/CCF composite by the simultaneous impregnation method to print; they put fibers in methyl dichloride solution with $8 \mathrm{wt} \%$ of PLA before print process. Printed samples had low surface quality and the radius of the fibers at the corners was clear. Yang et al. [26] investigated the mechanical and tensile properties of ABS/CCF by the simultaneous impregnation system. Printed samples were in the shell form. Dickson et al. [27] evaluated the tensile and flexural properties of Nylon/continuous glass fiber (CGF), Nylon/CCF, and Nylon/Kevlar composites. Batini et al. [28] succeeded in producing PLA/aramid composite by simultaneously feeding the aramid fibers and PLA through the feeding section. Printed samples were in the shell form. Ishii et al. [29] investigated PA/CCF composites fracture behavior before and after bending test with different radiuses of curvatures. Lack of truly adjacent fibers was also seen in circular paths.

Ibrahim et al. [30] examined the tensile properties of metal/wire reinforced composites. Parandoush et al. [31] examined mechanical bending properties by using a 3D printer based on laminated object manufacturing technology with Prepeg composite sheets of CCF composites 
(PA6/CCF). Matsuzaki et al. [32] investigated the effect of curvature radius and fibers size on the accuracy in printing continuous fiber composites (PLA/CCF). Printed samples were circular and in the small number of layers. Akhoundi et al. [33] investigated the effect of layer height and printing width on fibers-volume percentage. They achieved different volume percentages (up to $49 \%$ ) for the PLA/CGF composites and also succeeded in printing composites (PLA +/CGF, PLA + wood/CGF, thermoplastic polyurethane (TPU)/CGF, polyoxymethylene (POM)/CGF, PA/CGF) with the fiber-volume percentage of about $30 \%$ and evaluating their tensile mechanical properties [2]. Heidari-Rarani et al. [34] investigated the tensile and flexural properties of PLA/CCF composites. The quality of printed samples was very poor in terms of appearance, dimensional accuracy and surface smoothness. Kousiatza et al. [35] monitored the temperature profile and residual strain for nylon/CGF and nylon/CCF composites with different printing patterns $\left(0, \pm 45\right.$ and $\left.90^{\circ}\right)$ by optical fiber Bragg grating sensors. Li et al. [36] quantitatively analyzed the tensile strength of the PLA/CCF composite. Dutra et al. [37] investigated several mechanical properties (longitudinal and transverse strength and modulus, longitudinal and compressive strength and shear modulus) of the Nylon/CCF composites. The printed specimens were standard specimens of tensile test (dog-bone), and the fiber radius at the corners was clear. An and $\mathrm{Yu}$ [38] printed new fiber reinforced continuous composite with shape memory matrix (PLA/CCF) to evaluate mechanical properties of samples. Mei et al. [39] investigated the mechanical properties of Nylon/CGF, Nylon/CCF and Nylon/Kevlar composites printed with various filling patterns. Zhang et al. [40] examined the tensile and flexural strength of PLA/CCF and Nylon/CCF composites. Printed specimens had poor dimensional accuracy and surface smoothness. Todoroki et al. [41] studied the tensile and shear strength properties of Nylon/CCF composites printed with $0, \pm 45$ and $90^{\circ}$ filling patterns. Lack of dimensional accuracy and low quality was observed in the corners of the printed samples.

In most of the previous studies, the printed samples had simple geometric shapes with certain radiuses at the corners and the surface quality of samples was often undesirable. In addition, products were often in the form of shells due to the big challenge of printing solid parts with sharp corners. It is necessary to have a complete understanding of FFF process and the proper adjustment of its pertinent parameters for printing desirable continuous fiber composites. The main parameter that affects this process is the feed of filament, which must be reduced when using continuous fibers and this parameter was discussed in previous studies [2,33]. The next important parameter is the printing speed, which affects the process and the quality of final products, especially at the corners. Therefore, the purpose of this study is to investigate the printing speed in FFF process to obtain high-quality products.

\section{Methodology}

\subsection{Hypothesis}

In printing composites with continuous fibers, there is always a solid fiber in the nozzle outlet which is not affected by the melting and solidification process of the polymeric matrix. In this section, a hypothesis for examining the printing speed for composites is presented as follows:

- When continuous fibers are present at the nozzle outlet, there is a speed limitation to perform the correct printing process because of the nozzle movement from end of a path to new one. At critical point, the solidification process of the polymer at the end of the previous path must be completed, and then the nozzle can start the new printing path. Otherwise, due to changes in the speed gradient, it is possible to observe a separated fiber or form an undesirable radius at the path corner.

- The deposited raster temperature in one path must be below the melting or glass transition temperature in order to properly print the next path. Also, it is important to calculate the temperature variations of the deposited raster and examine their relations with the printing speed, which is expressed in the G-code system after the letter F in millimeters per minute.

\subsection{Heat transfer in FFF process}


Based on Figure 1 that adapted from [11], it is supposed that a raster with a specified length deposits on the bed and then an element is chosen to calculate the temperature at various points by thermal analysis. We suppose that the cross-section of the element and deposited raster are like a rectangle. So, we skip the corners of round raster because they don't have noticeable influence on the cross-section size and analysis [11].

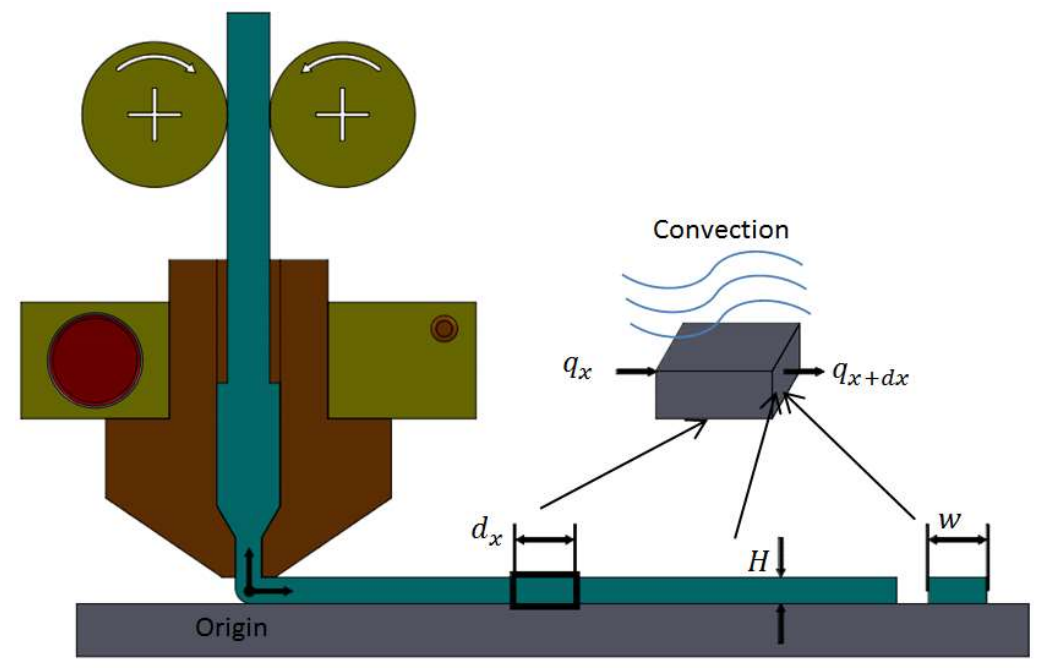

Figure 1. The element for thermal analysis.

Indeed, it is presumed that the coordinate origin is at the nozzle outlet, and the heat transfer is one-dimensional along the x-axis. The temperature is defined at the starting point of the process and can be measured after a certain distance. Obviously, the element length is equal to $d x$, the element width is equivalent to the extrusion width $(w)$ and the element thickness is equal to the layer height (h). The heat transfer from the element bottom to the bed (or the prior layer) is equal to the convection heat transfer by the environment, so according to the first law of thermodynamics for this element [11]:

$$
q_{x}-q_{x+d x}-h_{\text {Conv }} A_{C o n v}\left(T-T_{\infty}\right)=\rho C V(d T / d t)
$$

Where $q_{x}, q_{x+d x}, h_{\text {Conv }}, A_{\text {Conv }}, T, T_{\infty}, \rho, C, V$ and $d T / d t$ donote the input conduction heat transfer to the element, the output conduction heat transfer from the element, convection heat transfer coefficient for the sections that are in contact with the environment, temperature of the specimen at a certain point, ambient temperature, density, the specific heat capacity, the volume of the element and the temperature change per time, respectively. If the temperature at $x=0$ equals to the nozzle temperature $\left(T_{N}\right)$, by assuming a certain distance from the nozzle, the solution to Equation (1) is [11]:

$$
T(x)=\left(T_{N}-T_{\infty}\right) e^{(x / 2)\left(a-\sqrt{a^{2}+4 b}\right)}+T_{\infty}
$$

In this equation:

$$
\begin{gathered}
a=\frac{\rho C v}{k} \\
b=\left(2 h_{\text {Conv }}(h+w)\right) / k w h
\end{gathered}
$$

$v$ and $k$ indicate printing speed and conduction heat transfer coefficient respectively. If there is a fiber with $d_{f}$ diameter at the nozzle outlet, and layers have a height equal to the fiber diameter, the values of $a$ and $b$ in Equation (2) are:

$$
\begin{gathered}
a=\frac{\rho C v}{k} \\
\left.b=\left(2 h_{\text {Conv }}(h+w)\right) / k\left(w h-(\pi / 4) d_{f}^{2}\right)\right)
\end{gathered}
$$


5 of 15

\subsection{Speed calculation with continuous fibers}

The maximum printing speed is a function of the extrusion width, the layer height, the nozzle temperature, the filament material, and the feed of the filament in FFF process. The print speed must be such that the raster can deposit properly on the bed. In general, 3D printers have a speed limitation based on stepper motors, and most machines' maximum speed is $400 \mathrm{~mm} / \mathrm{s}$, which can print properly at high temperatures and low layer heights. If Equation (2) is solved at different speeds, the resulting graphs show the temperature drop in terms of the distance from the nozzle at various speeds. Also, if the length of each printing path is constant, the maximum printing speed can be determined according to the melting or glass transition temperature of the polymer and other printing conditions.

\subsection{Materials and equipment}

In this research, the FFF Quantum 2020 3D printer made in Iran was used (with $195 \times 195 \times 200$ $\mathrm{mm} 3$ building dimensions), which was modified in its nozzle for printing composite materials, the details were given in [19]. PLA filament with diameter of $1.75 \mathrm{~mm}$, provided from Digimaker, Canada, was employed as the polymer feedstock material for 3D printing. E-glass fiber Yarn was supplied by Jiahe Taizhou Co. (China), with the mass per length of $0.1 \mathrm{~g} / \mathrm{m}$. The Dino-Lite digital microscope (Taiwan) with a magnification of $200 X$ was used to detect the fibers yarn placement in the matrix and measuring the radius of corners.

\section{Results and Discussion}

\subsection{Calculating printing speed}

Using the values in Table 1 for the PLA matrix, the solution of Equation (2) is:

$$
T(x)=193 e^{-10.194 x}+27
$$

Table 1. property of the polymeric material and the value of the process parameters $[42,43]$.

\begin{tabular}{cc}
\hline Parameter & Value \\
\hline Nozzle Temperature & $T_{N}=220^{\circ} \mathrm{C}$ \\
Ambient Temperature & $T_{\infty}=27^{\circ} \mathrm{C}$ \\
Print Speed & $v=0.04 \mathrm{~m} / \mathrm{s}$ \\
Density & $\rho=1250 \mathrm{Kg} / \mathrm{m}^{3}$ \\
Convection Heat Transfer Coefficient & $h_{\text {Conv }}=75 \mathrm{w} / \mathrm{m}^{2}{ }^{\circ} \mathrm{C} \quad[43]$ \\
Specific Heat Capacity & $C=2060 \mathrm{j} / \mathrm{Kg}^{\circ} \mathrm{C}[44]$ \\
Thermal Conductivity Coefficient & $k=0.195 \mathrm{w} / \mathrm{m}^{\circ} \mathrm{C}[44]$ \\
Layer Height & $h=0.2 \mathrm{~mm}$ \\
Extrusion Width & $w=0.5 \mathrm{~mm}$ \\
Fiber Volume Percentage & $31.5 \%$ \\
Feed of Filament & $0.685[3]$
\end{tabular}

Figure 2 shows the diagram of temperature changes in deposited raster versus the nozzle motion. It is evident that when the nozzle goes away, the filament temperature decreases. By using the data in Table1 and assuming continuous glass fibers with a diameter of $0.2 \mathrm{~mm}$ at the nozzle outlet, the final solution of the Equation (4) is:

$$
T(x)=193 e^{-14.863}+27
$$

Figure 2 shows the temperature changes in the deposited raster. 


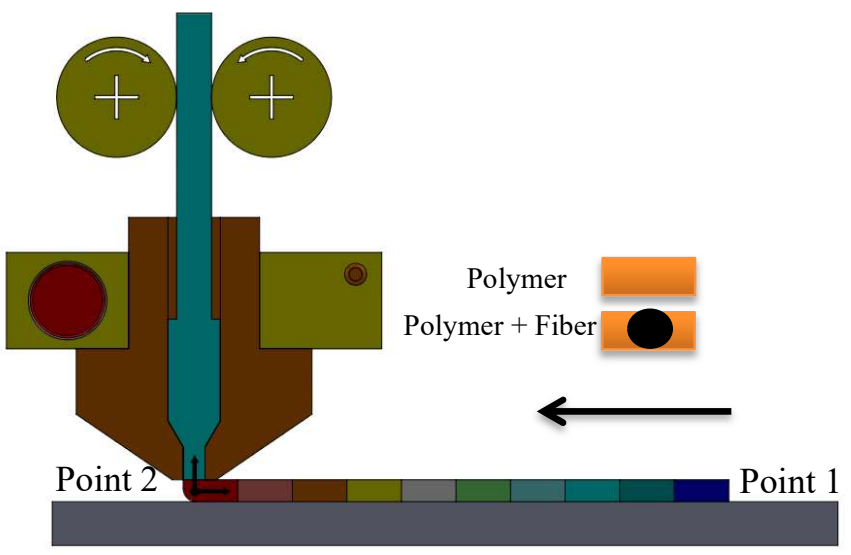

$\mathrm{a}$

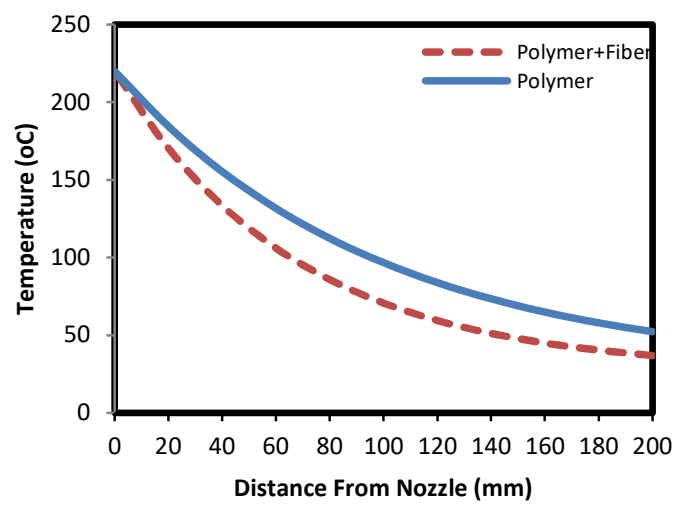

b

Figure 2. a) Temperature changes in the deposited raster. b) Effect of the travel distance from the nozzle on the temperature of the composite and non-composite deposited raster.

As shown in Figure 2a, the nozzle starts at point 1, and prints a distance of $200 \mathrm{~mm}$. As the nozzle moves, the temperature of the deposited filament changes according to Figure $2 b$ for the neat polymer and the composite sample. It is observed that in presence of the fibers, the heat transfer rate and the heat loss rises because of the reduction in the polymer. If Equation (2) is solved for different velocities $(4,40$ and $400 \mathrm{~mm} / \mathrm{s})$, Figure 3 is obtained using the data presented in Table 1 and continuous fibers with $0.2 \mathrm{~mm}$ diameter. The data in Table 1 is shown for PLA, and the glass transition and the melting temperature are $80^{\circ} \mathrm{C}$ and $180^{\circ} \mathrm{C}$, respectively. 


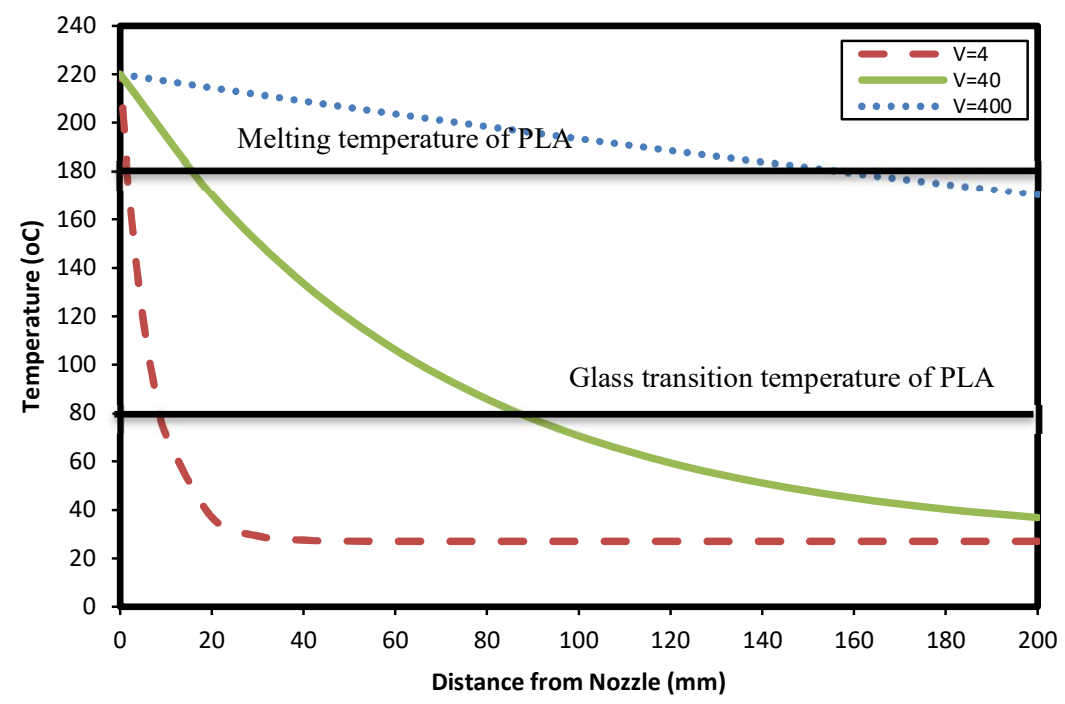

Figure 3. Temperature as a function of the travel distance from the nozzle at different print speeds.

As shown in Figure 3, at the low printing speed $(4 \mathrm{~mm} / \mathrm{s})$, the temperature drops below the glass transition or melting point in short paths (about under $20 \mathrm{~mm}$ ). When there is a direction change at the end of a printing path, the temperature must drop below the melting or glass transition temperature. At the end of the current path or at the beginning of the new one, the speed can be determined based on graphs such as those shown in Figure 3 in terms of path length.

For example, with a printing temperature of $220^{\circ} \mathrm{C}$ and printing speed of $40 \mathrm{~mm} / \mathrm{s}$ for a direct path, the temperature reaches below the melting line if the distance equals to $20 \mathrm{~mm}$ from the nozzle. By reducing speed to $4 \mathrm{~mm} / \mathrm{s}$ and assuming that the temperature is lower than the melting line, the distance equals to $4 \mathrm{~mm}$. So, it is necessary to drop the amount of printing speed at the beginning of a curved path to achieve printing process with excellent radius at corners. Also, with lower printing speed $(4 \mathrm{~mm} / \mathrm{s})$, the radius at the intersection of the two paths is minimized. Otherwise, without a reduction in the printing speed like in Figure 4 that adapted from [25], the intersection of the two paths have an undesirable radius, and higher printing speeds lead to greater radiuses.

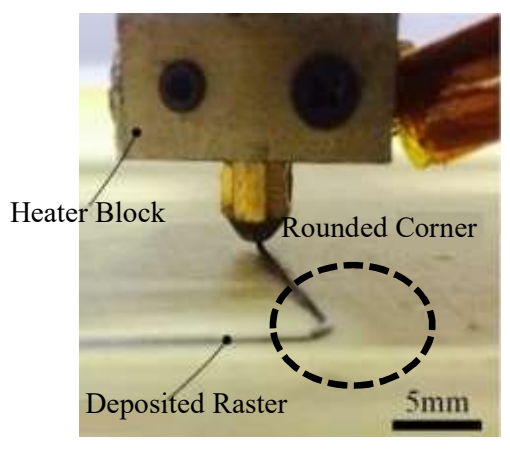

Figure 4. Creating rounded corners at a high printing speed.

The deceleration at the end of the current path and at the beginning of the next path is important for printing circular and arc paths because they are approximated by straight lines in FFF printers. Therefore, for printing curved and circular paths with continuous fibers to perform the printing process correctly, the printing speed must be determined based on approximated short paths. This estimation becomes more critical when the radius of the circle or arc decreases. 
8 of 15

\subsection{Effect of fiber volume percentage on printing speed}

Using the data in Table 1 and the fiber diameter of $0.2 \mathrm{~mm}$, the fiber volume percentage in Equation 6 and Figure 3 equals 31.5\% based on Equation 7 [2].

$$
V_{f}=\left(A_{f}\right) /(w h)
$$

According to previous studies, the maximum volume of fibers obtained through this method is $50 \%$ [2] by deeming changes in the volume of fibers resulting from changes in the extrusion width. Figure 5 is achieved by solving Equations 2 for the data in Table 1 on the basis of different extrusion width (which represents different volume percentages).

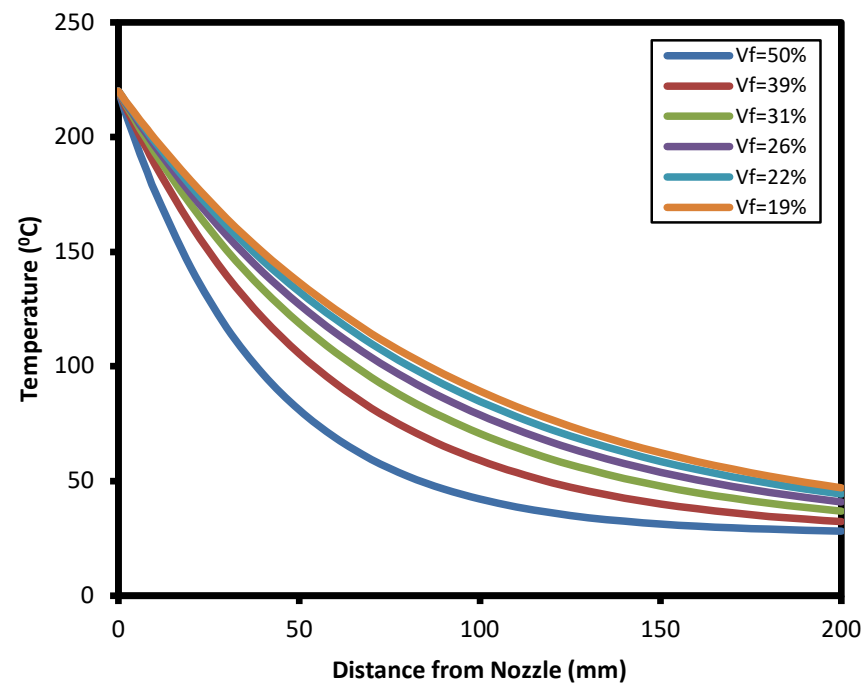

Figure 5. Temperature variations as a function of distance from the nozzle at different fiber volume percent.

Figure 5 demonstrates that at a constant printing speed, the temperature' drop rate is increased when there is more fiber in the nozzle outlet (growth in the fiber volume) because of reduction in polymer material.

\subsection{Experimental tests}

This section investigates the effect of printing speed on the sample' quality and the radii created at the corners. At first, some squared specimens are printed at $50 \mathrm{~mm}$ sides with different printing speeds $(2,4,6,8,10,15$, and $20 \mathrm{~mm} / \mathrm{s})$ and various parameters mentioned in Table 1, and then the radii of their corners are measured. Table 2 shows the mean radius created at the corners and the printing time of each sample for all printing speeds. The values are presented for five samples and mean the results are reported.

Table 2. Corner radius values and printing time for different constant speeds.

\begin{tabular}{ccc}
\hline Printing speed $(\mathbf{m m} / \boldsymbol{s})$ & Corner radius $(\mathbf{m m})$ (average) & Printing time $(\boldsymbol{s})$ \\
\hline 2 & 1.68 & 490 \\
4 & 1.97 & 255 \\
6 & 3.28 & 177 \\
8 & 3.55 & 138 \\
10 & 5.52 & 114 \\
15 & 9.24 & 83 \\
20 & Failure of print & 67 \\
\hline
\end{tabular}

As it is evident that when printing speed increases, the radius created at the corners has an upward trend in contrast with printing time. Figure 6 shows the pictures of printed samples; only 
the samples printed at 2 and $4 \mathrm{~mm} / \mathrm{s}$ have the correct shape, the radius value is high at 6 and 8 $\mathrm{mm} / \mathrm{s}$ and is not desirable at 10 and $15 \mathrm{~mm} / \mathrm{s}$.

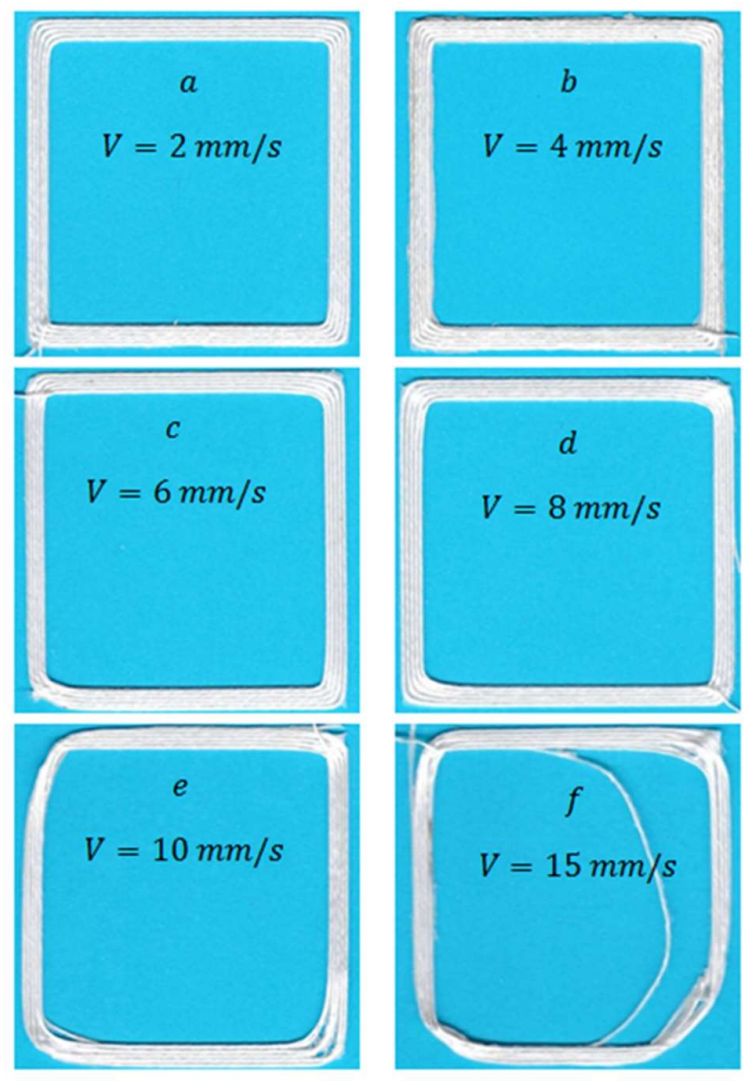

Figure 6. Printed samples at printing speeds of a) 2, b) 4, c) 6, d) 8, e) 10 and f) $15 \mathrm{~mm} / \mathrm{s}$

If the intersection point of $4 \mathrm{~mm} / \mathrm{s}$ (and lower velocities if plotted) and the PLA melting point are considered in Figure 3, it can be seen that in paths less than $2 \mathrm{~mm}$ the temperature of the polymer falls below the melting point and the solid polymer prevents the formation of fiber radius at the corners. Therefore, at printing speeds of less than $4 \mathrm{~mm} / \mathrm{s}$, the radius created at the corners is less. As the print speed increases (for example, $10 \mathrm{~mm} / \mathrm{s}$ ), the filament reaches below the melting point over a distance of $10 \mathrm{~mm}$. Then if the nozzle direction changes, the fibers are separated from the polymer because of the not frozen polymer and the tension of the fibers. So, the radius of the fibers becomes larger at the corners.

According to Figure 7, the speed decreases in the specified areas based on the hypothesis described in the 3.1 section.
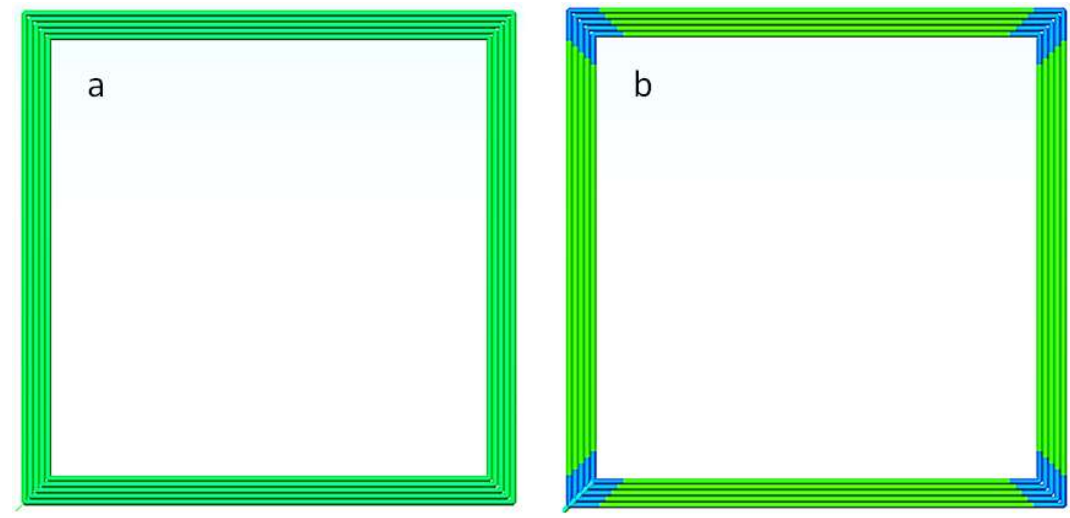

Figure 7. Printing samples at a) constant and b)variable speeds in corners. 
The printing speed in these samples is $15 \mathrm{~mm} / \mathrm{s}$ (the maximum speed for printed samples) at direct paths, and $0.1,0.25,0.5,1$ and $2 \mathrm{~mm} / \mathrm{s}$ are chosen for highlighted areas. Table 3 shows the mean radius vreated at the corners and the printing time of each sample for various printing speeds. The values are presented for the five samples and the mean results are reported.

Table 3. Corner radius and print time values for samples with variable speed printing.

\begin{tabular}{ccc}
\hline Printing speed in corners $(\mathbf{m m} / \mathbf{s})$ & Corner radius $(\mathbf{m m})$ (average) & Printing time $(\boldsymbol{s})$ \\
\hline 0.1 & 1.37 & 878 \\
0.25 & 1.47 & 558 \\
0.5 & 1.56 & 318 \\
1 & 1.65 & 198 \\
2 & 1.87 & 138 \\
\hline
\end{tabular}

With the decrease in printing speed at the corners, there is drop in the turn radius rapidly, but the reduction isn't considerable. Moreover, the printing time can be significantly reduced. The pictures of correctly printed samples are shown in the Figure 8.

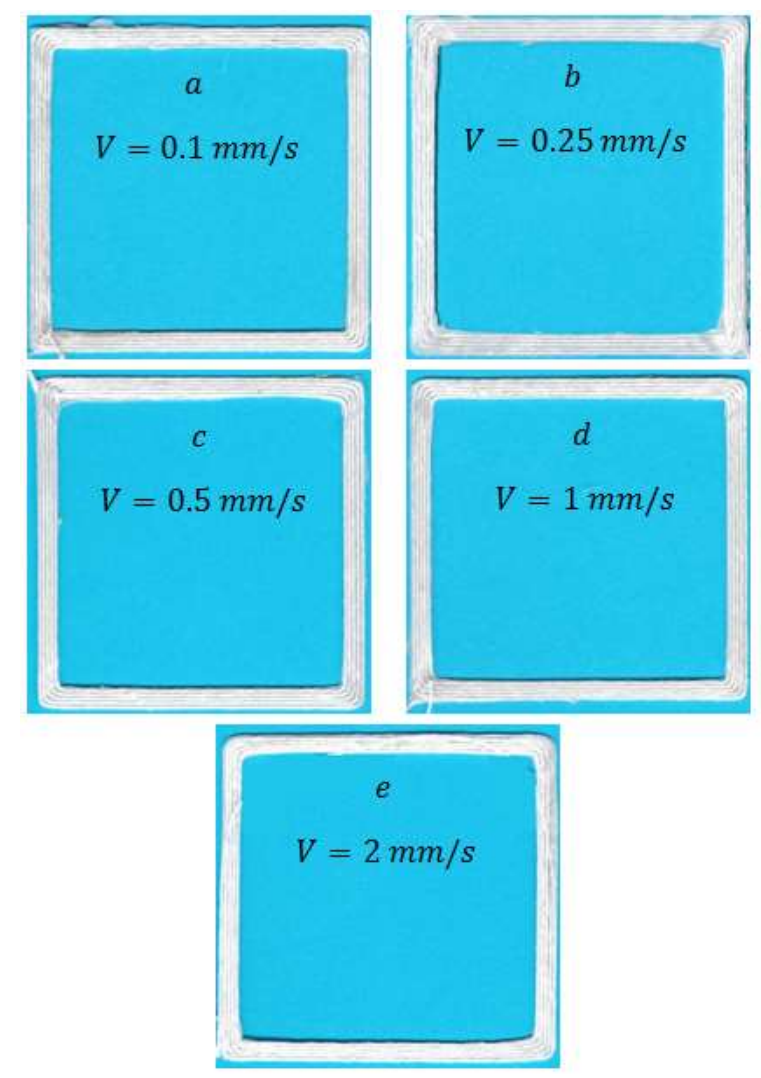

Figure 8. Printed samples at printing speeds of a) 0.1 , b) 0.25 , c) 0.5 , d) 1 , and e) $2 \mathrm{~mm} / \mathrm{s}$ at corners and $15 \mathrm{~mm} / \mathrm{s}$ at other paths.

Figure 9 a shows corner radius versus printing time and Figure $9 \mathrm{~b}$ demonstrates printing time versus printing time for samples with constant and variable speeds. According to the plots, with increase in printing speed at the corners, the radius of the corners has a similar trend in contrast with printing time. 

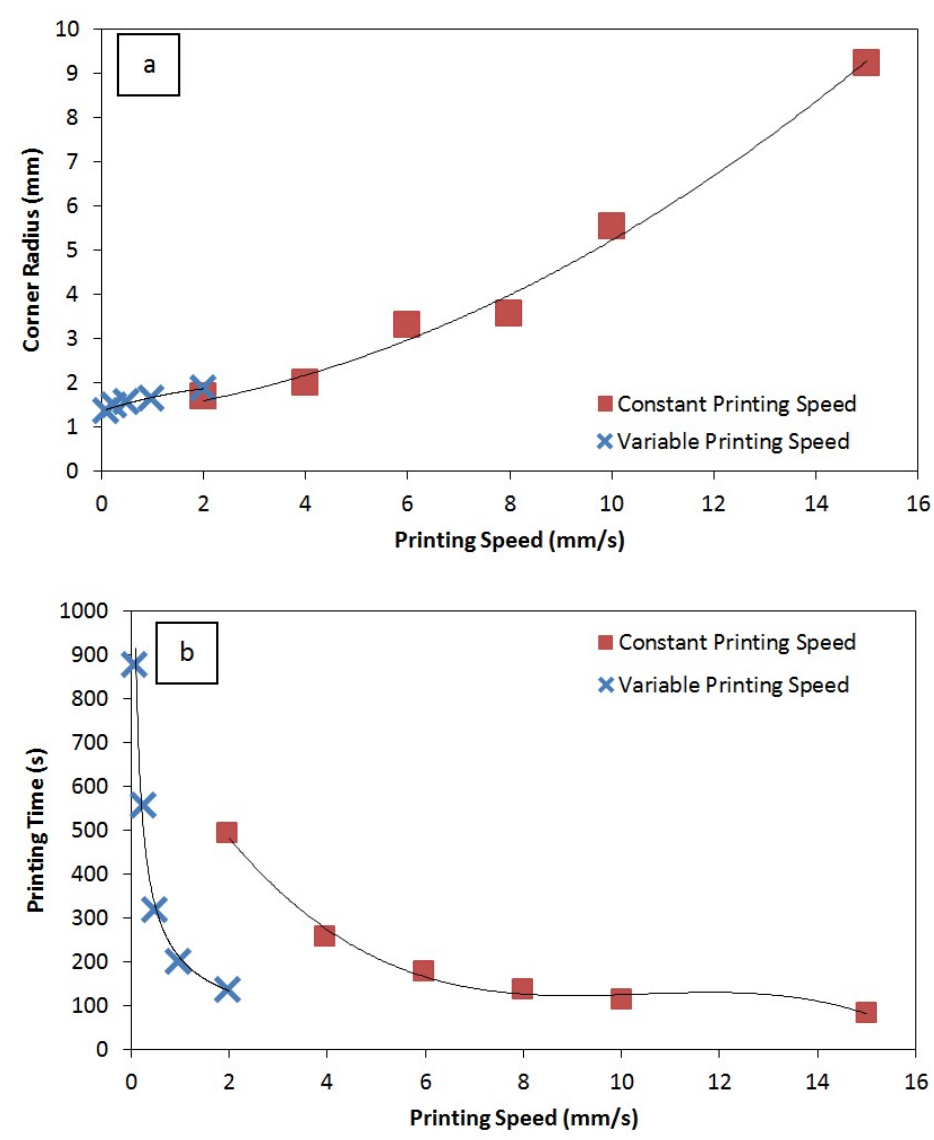

Figure 9. (a) Corner radius and (b) printing time variations according to printing speed.

As shown in Figure 9a, for specimens where the print speed is constant throughout the process, the amount of radius created at the corners increases as the print speed grows. If the speed at the corners decreases, the radius of the corners decreases too with the same proportion. The reason is that when the printing speed decreases, the deposited raster has enough time to cool, which prevents the fibers from separating at the corners. It can also be seen in Figure $9 \mathrm{~b}$ that the printing time rises when the print speed decreases both in the samples with constant print speed and in the samples with variable speeds where the speed is slower at the corners. The main reason for increasing the printing time by reducing the speed is the reduction of axes' movements during the printing process.

It is clear that with increasing printing speed at the corners, the radius of the corners rises too and the minimum printing speed is observable at the corner of arcs, which in this study has a minimum radius of $1.37 \mathrm{~mm}$. Furthermore, modification of the program and the speed at the corners will result in desirable products.

\subsection{Sample production strategy}

In this research, the following algorithm is employed to modify the G-code and printing speed at the corners.

- Reading the program

- Calculating the path length of each program line where a layer is deposited. (Lines of the program beginning with G1 code)

- Defining the path length during the solidification process for the deposited raster. (L) 
- If the path length in each line of the program is greater than the specified length, that path must be divided into two paths; a path with length of $\mathrm{L}$ at the end and a path from beginning to the distance of $\mathrm{L}$ from it.

- Creating new paths

- Measuring new lengths

- Calculating the feed of filament for each path and put it after the letter $\mathrm{E}$ in each line of the program

- Creating a database of the speeds required for each path according to the length, process conditions and filament material

- Adding the speed based on the length of each path after the letter $\mathrm{F}$ in the related line.

- running the program

Using the strategy with a fiber cutting system is vital for manufacturing high-quality products. Figure 10 indicates some desirable printed parts with this strategy.

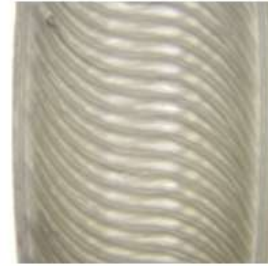

a

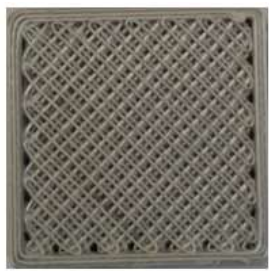

d

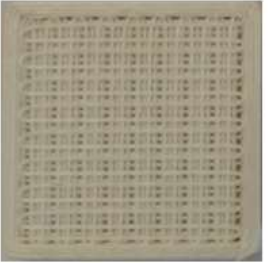

$\mathrm{b}$

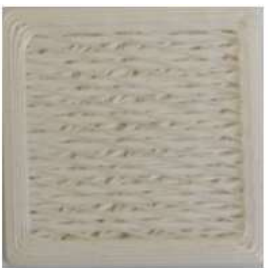

e
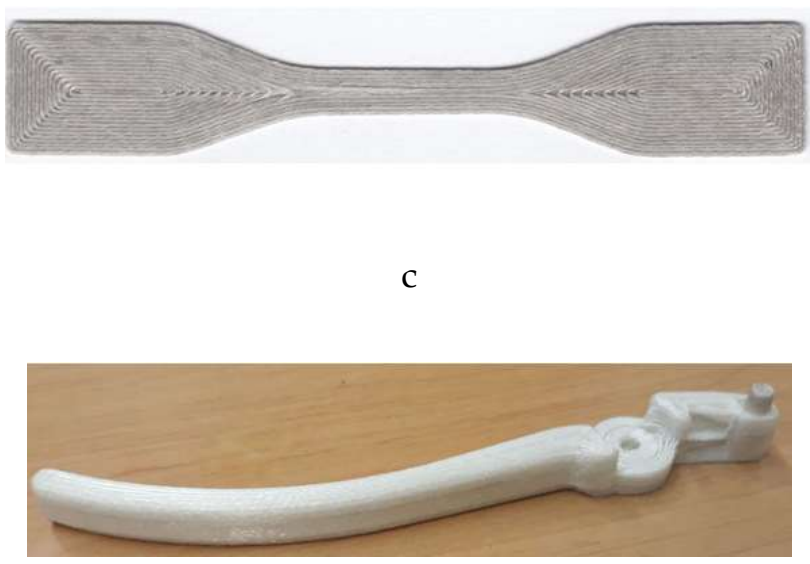

f

Figure 10. Printed parts with the proposed algorithm. a) cmposite sample with continuous fibers in the curved path, b) composite sample with continuous fibers $0 / 90, c$ ) standard specimens of tensile test (dog-bone) d) composite sample with continuous fibers \pm 45 , e) composite sample with continuous fibers $0 / 15 / 30 / 45 / 60 / 75 / 90 / 10 / 5 / 120$, and $f$ ) handle of a pair of pliers

\section{Conclusion}

In this study, the maximum printing speed for continuous fiber composites by the FFF process was determined. For this purpose, firstly, the relationship between the temperature changes in the deposited fiber was calculated, and then based on the presented hypothesis; the maximum printing speed was obtained for different paths. The hypothesis is that there is a speed limitation for the correct printing process when there is a continuous fiber in the nozzle outlet. The limitation emerges when the nozzle is moved from one end of the path to the new one, so the solidification process of the polymer material must occur at the end of the previous path and then the nozzle can move through the new one. Otherwise, due to changes in the speed gradient, separating of fibers or incorrect printing of corners is possible to happen. The assumed hypothesis presents the relationship between the radius of corners and printing speed, and as a result, the minimum printed radius is $1.37 \mathrm{~mm}$ at $0.1 \mathrm{~mm} / \mathrm{s}$ printing speed. Also, at a constant print speed, the radius of the corners increases with growing speed, and it is not possible to print the sample by increasing the speed from a certain limit (here $20 \mathrm{~mm} / \mathrm{s}$ ) that depends on the path length. Also, as the speed decreases, the radius of the corners falls too in contrast with the printing time. Therefore, to reduce the printing time, it is necessary to decrease only the printing speed at the corners where determining the speed is a function of the thermal properties of the material and the length of the depositing path. In this 
study, all calculations were for CGF/PLA composites. Finally, correct printed samples indicated the validity of the proposed hypothesis and strategy to achieve high-quality products with continuous fibers.

Author Contributions: Conceptualization, methodology, software, supervision and validation (Behnam Akhoundi; Faramarz Hajami); writing-original draft preparation (Mojtaba Nabipour; Amir Mosavi; Shahab S.; Shokri); experimental test (Omid Kordi).

Conflicts of Interest: The authors declare no conflicts of interest.

\section{References}

1. Bustillos, J.; Montero, D.; Nautiyal, P.; Loganathan, A.; Boesl, B.; Agarwal, A. Integration of graphene in poly(lactic) acid by 3D printing to develop creep and wear-resistant hierarchical nanocomposites. Polymer Composites 2018, 39, 3877-3888, doi:10.1002/pc.24422.

2. Nabipour, M.; Akhoundi, B.; Bagheri Saed, A. Manufacturing of polymer/metal composites by fused deposition modeling process with polyethylene. Journal of Applied Polymer Science 2020, 137, 48717.

3. Nabipour, M.; Akhoundi, B. An experimental study of FDM parameters effects on tensile strength, density, and production time of $\mathrm{ABS} / \mathrm{Cu}$ composites. Journal of Elastomers \& Plastics 2020, 0, 0095244320916838, doi:10.1177/0095244320916838.

4. Papon, E.A.; Haque, A. Review on process model, structure-property relationship of composites and future needs in fused filament fabrication. Journal of Reinforced Plastics and Composites 2020, 0, 0731684420929757, doi:10.1177/0731684420929757.

5. Liu, W.; Li, Y.; Liu, B.; Wang, G. Development of a novel rectangular-circular grid filling pattern of fused deposition modeling in cellular lattice structures. The International Journal of Advanced Manufacturing Technology 2020, 10.1007/s00170-020-05461-0, doi:10.1007/s00170-020-05461-0.

6. Shaffer, S.; Yang, K.; Vargas, J.; Di Prima, M.A.; Voit, W. On reducing anisotropy in 3D printed polymers via ionizing radiation. Polymer 2014, 55, 5969-5979.

7. Akhoundi, B.; Nabipour, M.; Hajami, F.; Shakoori, D. An Experimental Study of Nozzle Temperature and Heat Treatment (Annealing) Effects on Mechanical Properties of High-Temperature Polylactic Acid in Fused Deposition Modeling. Polymer Engineering \& Science 2020, 60, 979-987, doi:10.1002/pen.25353.

8. Mishra, D.K.; Pandey, P.M. Experimental investigation into the fabrication of green body developed by micro-extrusion-based 3D printing process. Polymer Composites 2020, n/a, doi:10.1002/pc.25514.

9. Murphy, C.A.; Collins, M.N. Microcrystalline cellulose reinforced polylactic acid biocomposite filaments for 3D printing. Polymer Composites 2018, 39, 1311-1320, doi:10.1002/pc.24069.

10. Saed, A.B.; Behravesh, A.H.; Hasannia, S.; Ardebili, S.A.A.; Akhoundi, B.; Pourghayoumi, M. Functionalized poly l-lactic acid synthesis and optimization of process parameters for 3D printing of porous scaffolds via digital light processing (DLP) method. Journal of Manufacturing Processes 2020, 56, 550-561.

11. Akhoundi, B.; Behravesh, A.H. Effect of Filling Pattern on the Tensile and Flexural Mechanical Properties of FDM 3D Printed Products. Experimental Mechanics 2019, 59, 883-897, doi:10.1007/s11340-018-00467-y.

12. Nabipour, M.; Behravesh, A.H.; Akhoundi, B. Effect of printing parameters on Mechanical Strength of Polymer-Metal composites Printed via FDM 3D printer. Modares Mechanical Engineering 2017, 17, 145-150.

13. Hedayati, S.K.; Behravesh, A.H.; Hasannia, S.; Saed, A.B.; Akhoundi, B. 3D printed PCL scaffold reinforced with continuous biodegradable fiber yarn: A study on mechanical and cell viability properties. Polymer Testing 2020, 83, 106347. 
14. Spoerk, M.; Savandaiah, C.; Arbeiter, F.; Sapkota, J.; Holzer, C. Optimization of mechanical properties of glass-spheres-filled polypropylene composites for extrusion-based additive manufacturing. Polymer Composites 2019, 40, 638-651, doi:10.1002/pc.24701.

15. Kabir, S.F.; Mathur, K.; Seyam, A.-F.M. A critical review on 3D printed continuous fiber-reinforced composites: history, mechanism, materials and properties. Composite Structures 2019, 111476.

16. Zhang, Y.; Li, H.; Yang, X.; Zhang, T.; Zhu, K.; Si, W.; Liu, Z.; Sun, H. Additive manufacturing of carbon nanotube-photopolymer composite radar absorbing materials. Polymer Composites 2018, 39, E671-E676, doi:10.1002/pc.24117.

17. O'Connor, H.J.; Dowling, D.P. Low-pressure additive manufacturing of continuous fiber-reinforced polymer composites. Polymer Composites 2019, 40, 4329-4339, doi:10.1002/pc.25294.

18. Ansari, M.Q.; Redmann, A.; Osswald, T.A.; Bortner, M.J.; Baird, D.G. Application of thermotropic liquid crystalline polymer reinforced acrylonitrile butadiene styrene in fused filament fabrication. Additive Manufacturing 2019, 29, 100813.

19. Akhoundi, B.; Behravesh, A.H.; Bagheri Saed, A. An innovative design approach in three-dimensional printing of continuous fiber-reinforced thermoplastic composites via fused deposition modeling process: In-melt simultaneous impregnation. Proceedings of the Institution of Mechanical Engineers, Part B: Journal of Engineering Manufacture 2020, 234, 243-259, doi:10.1177/0954405419843780.

20. Mori, K.-i.; Maeno, T.; Nakagawa, Y. Dieless forming of carbon fibre reinforced plastic parts using 3D printer. Procedia Engineering 2014, 81, 1595-1600.

21. Van Der Klift, F.; Koga, Y.; Todoroki, A.; Ueda, M.; Hirano, Y.; Matsuzaki, R. 3D printing of continuous carbon fibre reinforced thermo-plastic (CFRTP) tensile test specimens. Open Journal of Composite Materials $2015,6,18$.

22. Matsuzaki, R.; Ueda, M.; Namiki, M.; Jeong, T.-K.; Asahara, H.; Horiguchi, K.; Nakamura, T.; Todoroki, A.; Hirano, Y. Three-dimensional printing of continuous-fiber composites by in-nozzle impregnation. Scientific reports 2016, 6, 23058.

23. Tian, X.; Liu, T.; Yang, C.; Wang, Q.; Li, D. Interface and performance of 3D printed continuous carbon fiber reinforced PLA composites. Composites Part A: Applied Science and Manufacturing 2016, 88, 198-205.

24. Melenka, G.W.; Cheung, B.K.; Schofield, J.S.; Dawson, M.R.; Carey, J.P. Evaluation and prediction of the tensile properties of continuous fiber-reinforced 3D printed structures. Composite Structures 2016, 153, 866-875.

25. Li, N.; Li, Y.; Liu, S. Rapid prototyping of continuous carbon fiber reinforced polylactic acid composites by 3D printing. Journal of Materials Processing Technology 2016, 238, 218-225.

26. Yang, C.; Yang, C.; Tian, X.; Tian, X.; Liu, T.; Liu, T.; Cao, Y.; Cao, Y.; Li, D.; Li, D. 3D printing for continuous fiber reinforced thermoplastic composites: mechanism and performance. Rapid Prototyping Journal 2017, 23, 209-215.

27. Dickson, A.N.; Barry, J.N.; McDonnell, K.A.; Dowling, D.P. Fabrication of continuous carbon, glass and Kevlar fibre reinforced polymer composites using additive manufacturing. Additive Manufacturing 2017, 16, 146-152.

28. Bettini, P.; Alitta, G.; Sala, G.; Di Landro, L. Fused Deposition Technique for Continuous Fiber Reinforced Thermoplastic. Journal of Materials Engineering and Performance 2017, 26, 843-848.

29. Ishii, K.; Todoroki, A.; Mizutani, Y.; Suzuki, Y.; Koga, Y.; Matsuzaki, R.; Ueda, M.; Hirano, Y. Bending fracture rule for 3D-printed curved continuous-fiber composite. Advanced Composite Materials 2019, 28, 383-395. 
30. Ibrahim, Y.; Melenka, G.W.; Kempers, R. Fabrication and tensile testing of 3D printed continuous wire polymer composites. Rapid Prototyping Journal 2018, 24, 1131-1141.

31. Parandoush, P.; Zhou, C.; Lin, D. 3D Printing of Ultrahigh Strength Continuous Carbon Fiber Composites. Advanced Engineering Materials 2019, 21, 1800622.

32. Matsuzaki, R.; Nakamura, T.; Sugiyama, K.; Ueda, M.; Todoroki, A.; Hirano, Y.; Yamagata, Y. Effects of set curvature and fiber bundle size on the printed radius of curvature by a continuous carbon fiber composite 3D printer. Additive Manufacturing 2018, 24, 93-102.

33. Akhoundi, B.; Behravesh, A.H.; Bagheri Saed, A. Improving mechanical properties of continuous fiber-reinforced thermoplastic composites produced by FDM 3D printer. Journal of Reinforced Plastics and Composites 2019, 38, 99-116.

34. Heidari-Rarani, M.; Rafiee-Afarani, M.; Zahedi, A. Mechanical characterization of FDM 3D printing of continuous carbon fiber reinforced PLA composites. Composites Part B: Engineering 2019, 175, 107147.

35. Kousiatza, C.; Tzetzis, D.; Karalekas, D. In-situ characterization of 3D printed continuous fiber reinforced composites: A methodological study using fiber Bragg grating sensors. Composites Science and Technology 2019, 174, 134-141.

36. Li, H.; Wang, T.; Joshi, S.; Yu, Z. The quantitative analysis of tensile strength of additively manufactured continuous carbon fiber reinforced polylactic acid (PLA). Rapid Prototyping Journal 2019.

37. Dutra, T.A.; Ferreira, R.T.L.; Resende, H.B.; Guimarães, A. Mechanical characterization and asymptotic homogenization of 3D-printed continuous carbon fiber-reinforced thermoplastic. Journal of the Brazilian Society of Mechanical Sciences and Engineering 2019, 41, 133.

38. An, Y.; Yu, W.-R. Three-dimensional printing of continuous carbon fiber-reinforced shape memory polymer composites. In Proceedings of AIP Conference Proceedings; p. 020008.

39. Mei, H.; Ali, Z.; Ali, I.; Cheng, L. Tailoring strength and modulus by 3D printing different continuous fibers and filled structures into composites. Advanced Composites and Hybrid Materials 2019, 2, 312-319.

40. Zhang, J.; Zhou, Z.; Zhang, F.; Tan, Y.; Yi, R. Molding process and properties of continuous carbon fiber three-dimensional printing. Advances in Mechanical Engineering 2019, 11, 1687814019835698.

41. Todoroki, A.; Oasada, T.; Mizutani, Y.; Suzuki, Y.; Ueda, M.; Matsuzaki, R.; Hirano, Y. Tensile property evaluations of $3 \mathrm{D}$ printed continuous carbon fiber reinforced thermoplastic composites. Advanced Composite Materials 2019, 1-16.

42. Bellehumeur, C.; Li, L.; Sun, Q.; Gu, P. Modeling of bond formation between polymer filaments in the fused deposition modeling process. Journal of manufacturing processes 2004, 6, 170-178.

43. Farah, S.; Anderson, D.G.; Langer, R. Physical and mechanical properties of PLA, and their functions in widespread applications-A comprehensive review. Advanced drug delivery reviews 2016, 107, 367-392. 